Recepción: 08 / 06 / 2018

Aceptación: 16 / 08 / 2018

Ciencias matemáticas

Publicación: 05 / 10 / 2018

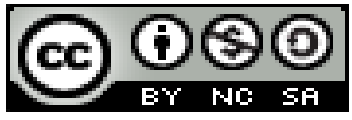

Artículo de investigación

\title{
Modelación Matemática para la estimación de tiempos en un proyecto utilizando MATLAB-Simulink
}

\section{Mathematical Modeling for the estimation of times in a project using MATLAB- Simulink}

\section{Modelagem Matemática para a estimativa de tempos em um projeto usando}

\section{MATLAB-Simulink}

Elvis Enrique-Arguello I

e_arguello@espoch.edu.ec

Wilson J. Villagrán-Cáceres II

wvillagran@espoch.edu.ec

Luis F. Buenaño-Moyano III

1fbuenanio@espoch.edu.ec

\section{Correspondencia: e_arguello@espoch.edu.ec}

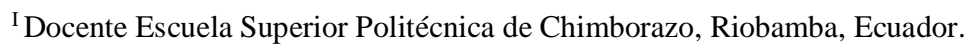

II Magister en Matemática Básica, Ingeniero en Electrónica y Computación, Tecnólogo en Informática Aplicada, Docente de la Escuela Superior Politécnica de Chimborazo, Riobamba, Ecuador.

III Magister en Gestión del Mantenimiento Industrial, Ingeniero Automotriz, Docente de la Escuela Superior Politécnica de Chimborazo, Riobamba, Ecuador.

${ }^{\text {IV }}$ Magister en Gerencia de la Educación Abierta, Licenciado en Ciencias de la Educación, Profesor de Enseñanza Media en la Especialización de Educación Física, Docente de la Escuela Superior Politécnica de Chimborazo, Riobamba, Ecuador.

\footnotetext{
${ }^{v}$ Magister en Gestión de la Calidad y Productividad, Ingeniero Industrial Mención Gestión de Procesos, Docente De La Escuela Superior Politécnica De Chimborazo, Riobamba, Ecuador.
} 


\section{Resumen}

Para este trabajo se tomó en consideración la obtención del tiempo de duración de un proyecto de acuerdo con las actividades que son necesarias para lograr el objetivo de este, simulándolo en el paquete Simulink del software Matlab. Al automatizar mediante la ayuda del software se obtuvo resultados precisos y mucho más efectivos, en contraste de los que lo hiciera el gerente del proyecto de manera manual o utilizando un software para la gestión de proyectos.

Palabras clave: estimación de tiempo; software; Matlab; Simulink.

\section{Abstract}

For this work it was taken into consideration the obtaining of the duration of a project according to the activities that are necessary to achieve the objective of this, simulating it in the Simulink package of Matlab software. By automating the software, we obtained accurate and much more effective results, in contrast to those made by the project manager manually or using software for project management.

Key words: time estimation; software; Matlab; Simulink.

\section{Resumo}

Para este trabalho foi levado em consideração a obtenção da duração de um projeto de acordo com as atividades necessárias para atingir o objetivo deste, simulando-o no pacote Simulink do software Matlab. Ao automatizar o software, obtivemos resultados precisos e muito mais eficazes, em contraste com aqueles feitos pelo gerente de projeto manualmente ou usando software para gerenciamento de projetos.

Palavras chave: estimativa de tempo; software; Matlab; Simulink.

\section{Introducción}

Desarrollar un proyecto conlleva cierta cantidad de tiempo, el mismo que tiene una gran relevancia para que el contratista realice correctamente sus funciones. Basado en estimaciones de tiempo, el gerente del proyecto tendrá que tomar ciertas decisiones importantes relacionada a clientes, empleados, otros proyectos, y actividades complementarias. 
El incumplimiento de fechas previstas para la culminación de una actividad generara prejuicios importantes, tales como perdidas económicas, atraso de tiempos para actividades relacionadas, y algo aún más relevante, pérdida de la confianza del cliente y una mala reputación.

Por otro lado, un correcto manejo de tiempo provocará que se utilicen la mínima cantidad de recursos disponibles con el fin de aumentar la rentabilidad.

Como se puede apreciar, el beneficio neto que producirá un proyecto esta ligado estrechamente con el tiempo, ya sea por las horas que hemos proyectado utilizar en determinada actividad, como la demora que provocará en el inicio de nuevas actividades.

\section{Métodos}

Como es de conocimiento general, investigaciones complejas y desarrollo de proyectos pueden ser manejados efectivamente si el gerente del proyecto tiene los recursos para poder planificar y controlar los cronogramas y costos que el trabajo requiere para poder alcanzar sus objetivos planteados. (PYME Online, 2018)

Mientras se planifica un proyecto, varias preguntas siempre salen a flote, entre ellas: ¿Cómo se debería completar el trabajo?, ¿Cuánto debe durar? ¿Cuánto va a costar? (PYME Online, 2018)

Los gerentes a todo nivel están en la necesidad de mejorar sus técnicas en todas las etapas del proyecto de modo que:

- Se defina correctamente el trabajo a ser realizado

- Se desarrolle un cronograma y proyección de costos más realista basado en la planificación de utilización de recursos para realizar dicho trabajo

- Determinar donde los recursos deben ser aplicados de modo que se pueda optimizar el tiempo para alcanzar objetivos

- Identificar esas áreas que pueden generar retrasos o gastos excesivos a tiempo, de modo que se pueda aplicar correctivos

Los siguientes son herramientas o técnicas utilizadas en la gerencia de proyectos:

1. Diagrama de barras 
2. Diagrama de red

\section{Diagrama de Barras}

El diagrama de barras fue introducido por Henery Grantt por los años 1900. El diagrama de barras es una representación gráfica en dos dimensiones de un proyecto. Estas barras representan las actividades planeadas a ser controladas. Después de un tiempo este diagrama fue modificado a un mapa de hitos. Se debe tomar en cuenta que el diagrama de barras representa actividades, y el mapa de hitos está relacionado con los eventos de los cuales se denota ya sea el inicio o el final de una actividad (Pumnia \& Khandelwal, 2006, pág. 8).

\section{Diagrama de redes}

El diagrama de redes es una técnica que implica cierto avance para la planificación gerencial. Esta técnica se basa en la caracterización grafica para todos los proyectos, todo el trabajo que se dé hacer en pasos muy bien definidos. Por ejemplo, para construir una casa, los pasos previos serian, realizar planos (i), excavar (ii), colocar columnas y paredes (iii), y finalmente construir la loza (iv). El diagrama de redes aprovecha sus caracterizas al representar los pasos del proyecto de una manera gráfica y objetiva en forma de redes. (Pumnia \& Khandelwal, 2006, pp. 9-10)

Las técnicas de redes son llamadas por varios nombres como PERT, CP, UNETICS, LESS, TOPS y SCANS. Sin embargo, estos y otros sistemas han emergido desde los siguientes dos mayores sistemas de redes:

\section{PERT}

\section{CPM}

\section{PERT}

PERT proviene de "Program Evaluation and Review Technique", por sus siglas en ingles. Este método básicamente fue desarrollado por Navy Special Projects Office en cooperación con Booz, Allen y Hamilton, una firma de consultoría empresarial además de Lockheed Missile System División encargada de evaluar la factibilidad de los cronogramas existentes en el programa de misiles Polaris y para reportar su progreso (Keefer \& Verdini, 2015). 
El método PERT usa un diagrama de redes compuesto de eventos los cuales deben ser establecidos para que el programa alcance sus objetivos. Un evento es ese instante particular en el cual alguna parte específica del cronograma es alcanzada. Este indica el punto en el tiempo y no requiere ningún recurso. PERT utiliza un diagrama de red de eventos orientados en el cual los eventos sucesivos son conectados por medio de flechas. (Keefer \& Verdini, 2015)

El método PERT es recomendado para aquellos proyectos u operaciones que no son de naturaleza repetitiva o para aquellos proyectos en los cuales realizar una estimación precisa del tiempo resulta complicado. (Keefer \& Verdini, 2015)

\section{CPM}

CPM proviene de "Critical Path Method" es decir, método de la ruta crítica. Las redes CPM, en la totalidad del proyecto consiste en un número de trabajos claramente reconocibles $\mathrm{u}$ operaciones conocidas como actividades. Las actividades usualmente son operaciones que toman su determinado tiempo en levarlas a cabo, y en las cuales los recursos son usados. Las uniones entre actividades son conocidas como eventos. (Keefer \& Verdini, 2015)

El diagrama CPM generalmente e usado en proyectos de tipo repetitivos, o para aquellos proyectos en los cuales se puede realizar una estimación de tiempo bastante preciso para poder completar cada actividad, e inclusive para aquellos proyectos en los cuales se puede realizar una estimación de costos bastante aceptable. Por ejemplo, CPM es bastante utilizado en el área de construcción, pero no lo es para investigación y proyectos de desarrollo. (Keefer \& Verdini, 2015)

\section{Comparación entre PERT y CPM}

Como se mencionó anteriormente, PERT utiliza un diagrama de eventos orientados, mientras que CPM utiliza una red de actividades orientadas. Esto no significa que el sistema PERT no tenga actividades o que CPM no tenga eventos. (Keefer \& Verdini, 2015)

En PERT se enfatiza en los eventos dados, mientras que en CPM se enfatiza en las actividades dadas. Ambos PERT y CPM tienen muchas cosas en común. Sin embargo, difieren en lo siguiente: 
- En CPM, la estimación de tiempo para la conclusión de actividades es de gran precisión, mientras que en PERT no son tan definidas

- En CPM, la optimización del costo es marcada como gran prioridad. La duración para completar una actividad depende mucho en la optimización del tiempo. Este costo no es directamente proporcional al tiempo. Por otro lado, en PERT, este asume que el costo variará conforme avance el tiempo, por lo que se busca la optimización del tiempo de modo que los costos se reduzcan.

Cabe mencionar que con el tiempo el método PERT y CPM fueron utilizados en conjunto en un mismo diagrama de red con el fin de optimizar todo recursos.

\section{Estimación de Tiempo}

Tiempo es la variable más esencial y básica en la aplicación del PERT. Hemos visto que el PERT es mayoritariamente usado para investigación y proyectos de carácter único. En estos proyectos, existe poca certeza acerca del tiempo que requerirá completar varias actividades. La estimación exacta de tiempo para poder completar las actividades en la utilización de este método es bastante difícil. (Farnum \& Staton, 1987)

Por lo tanto, para poder realizar una estimación de tiempo aproximada, se realiza primero tres tipos de estimaciones:

La estimación optimista

La estimación pesimista

La estimación más probable

\section{Estimación optimista (a)}

Esta tiene la particularidad de ser muy poco probable, es la estimación mas corta de tiempo para lo cual la actividad pueda ser concluida, se toma en cuenta condiciones ideales. Esta estimación solo se dará si todo el proceso se desarrolla perfectamente, sin ninguna adversidad. (Farnum \& Staton, 1987) 


\section{Estimación pesimista (b)}

Es la apuesta a la mayor cantidad de tiempo que se le puede dedicar a una actividad para que sea completada. Se refiere a que se tomará ese tiempo siempre y cuando todo vaya mal, bajo condiciones anormales durante todo el proceso. Sin embargo, esta estimación no toma en cuenta retrasos por catástrofes inusuales tales como, terremotos, inundaciones, incendios. (Farnum \& Staton, 1987)

\section{Estimación más probable (m)}

La estimación más probable es el tiempo que, en la mente del gerente, representa el tiempo que una actividad tomara en ser concluida si condiciones normales prevalecen. Este tiempo se encuentra entre el optimista y el pesimista. Se considera condiciones normales, es decir no ocurre nada emocionante durante el proceso.

Finalmente, estos tres tiempos son tomados en cuenta para encontrar una estimación final del tiempo que nos llevará concluir una actividad mediante la siguiente formula:

$$
t=\frac{a+4 m+b}{6}
$$

\section{Simulación en MATLAB}

Para poder realizar la simulación competente se utilizó el paquete Simulink, mismo que nos permite representar de manera gráfica los procesos que se deben seguir para poder resolver este tipo de redes y obtener la media de tiempos y la ruta crítica.

Se puede implementar este proceso a un sinfín de proyectos, para fines de aplicación de la simulación se utilizará como ejemplo la introducción de un nuevo vehículo al mercado, el consta de las siguientes actividades: 
Cuadro 1: Introducción de un nuevo vehículo

\begin{tabular}{|l|l|l|l|l|l|}
\hline Actividad & Predecesor & Tiempos & Pesimista & Optimista & Más \\
probable
\end{tabular}

Fuente: Autor

Este es el modelo preliminar, antes de realizar la ejecución del problema:

Figura 1: Preliminar en Simulink

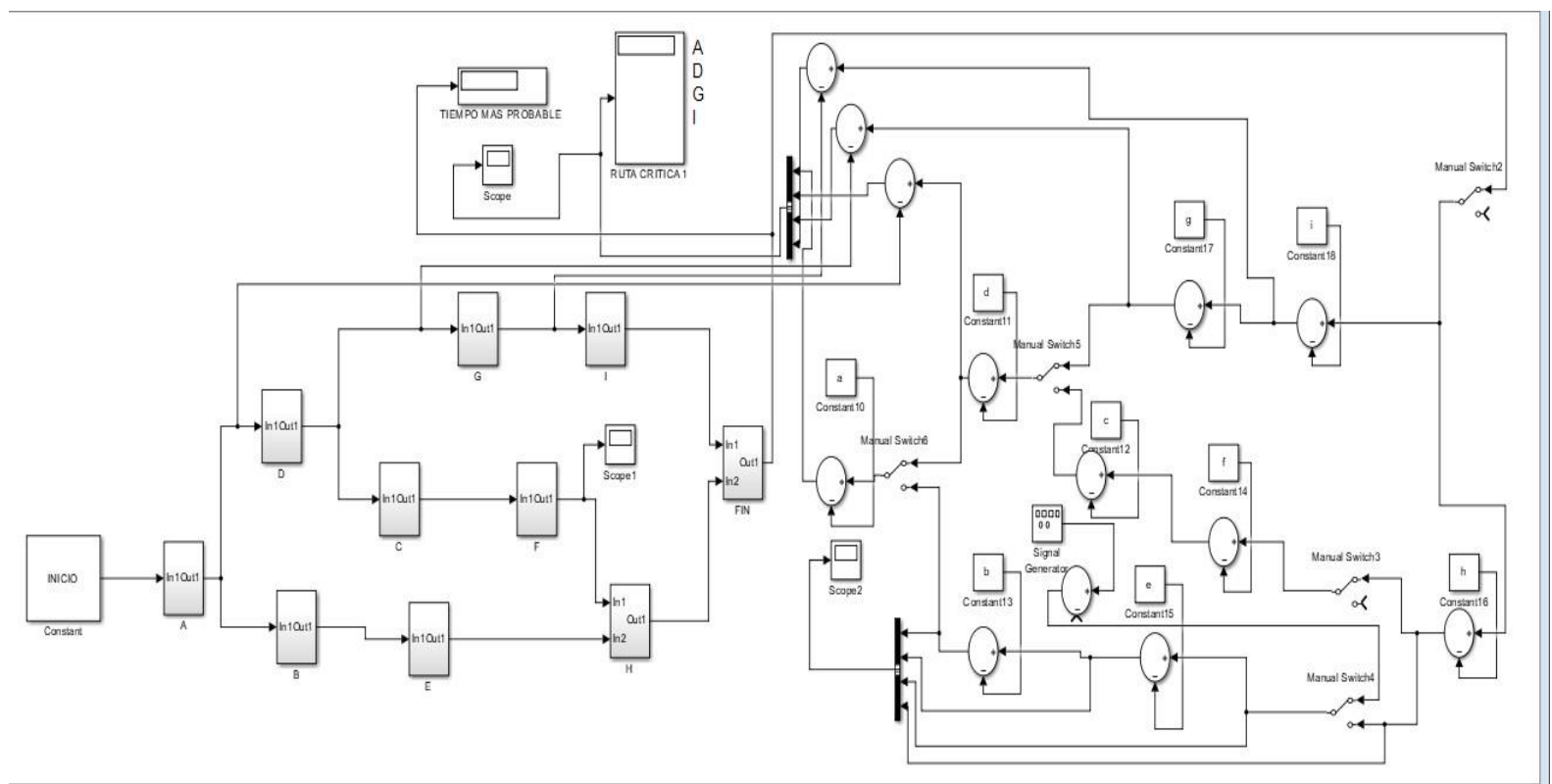

Fuente: Autor 
Una vez ejecutado el programa obtendremos los siguientes resultados:

Figura 2: Corrida del programa Simulink Resultante

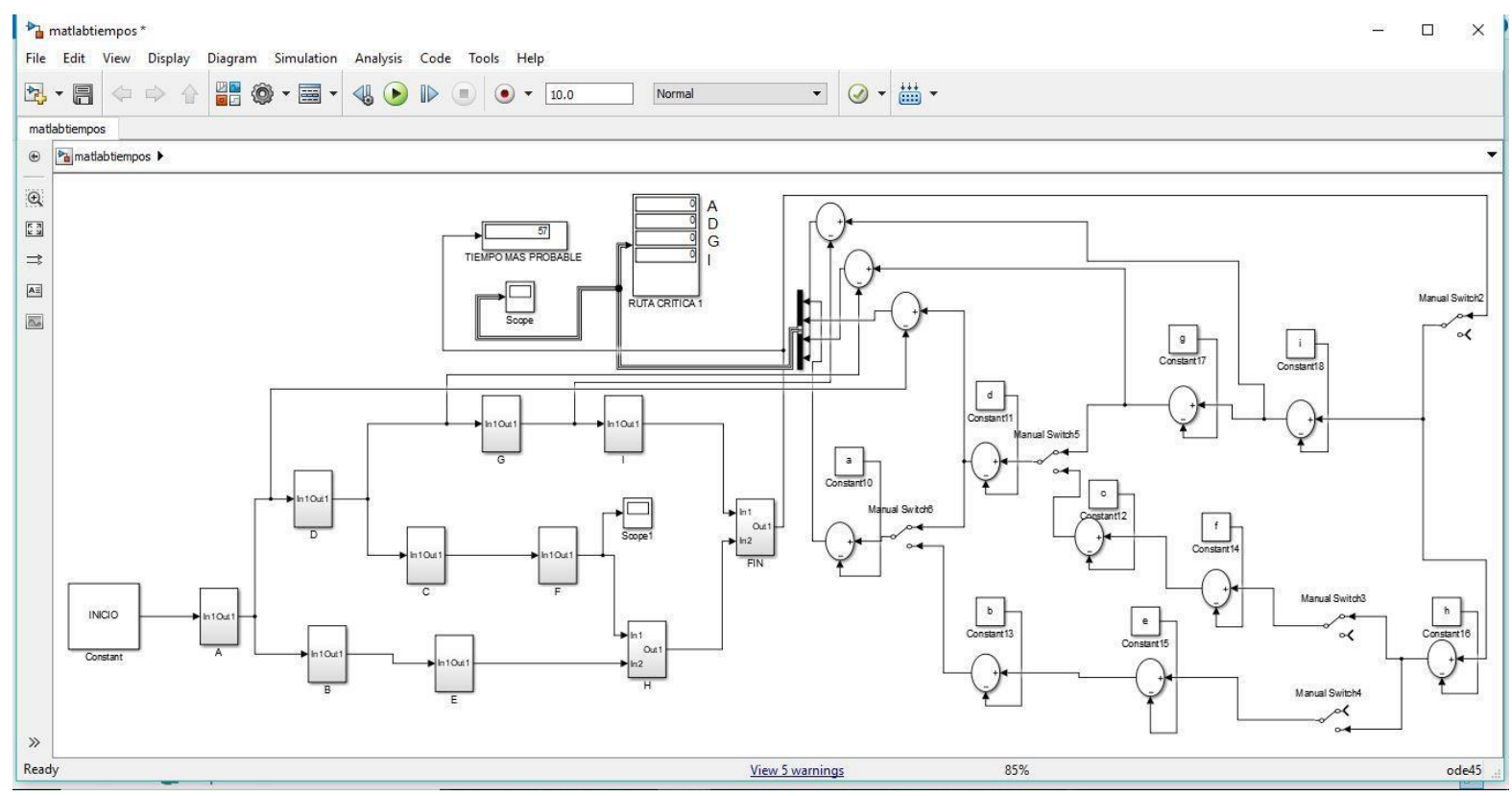

Fuente: Autor

De la cual se obtiene como gráficas del scoop lo siguiente:

Figura 3: Mejores Opciones para proyecto de acuerdo a Soop

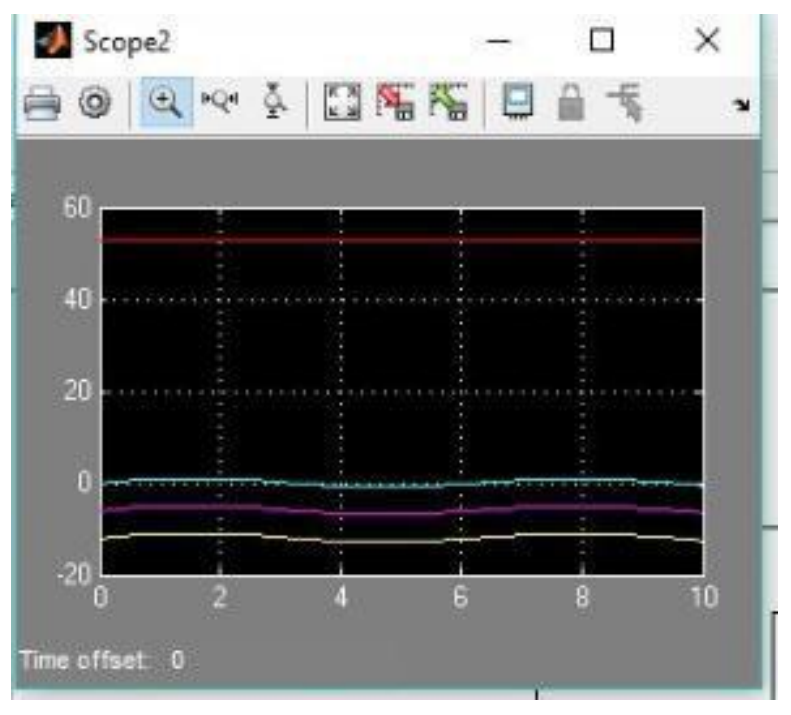

Fuente: Autor 
Resumiéndose cada una de las líneas en las diferentes rutas implementadas en proyecto.

\section{Resultados}

El tiempo de la ruta crítica es igual a 57, siendo está conformada por I, G, D, A

Figura 4: Ruta Crítica

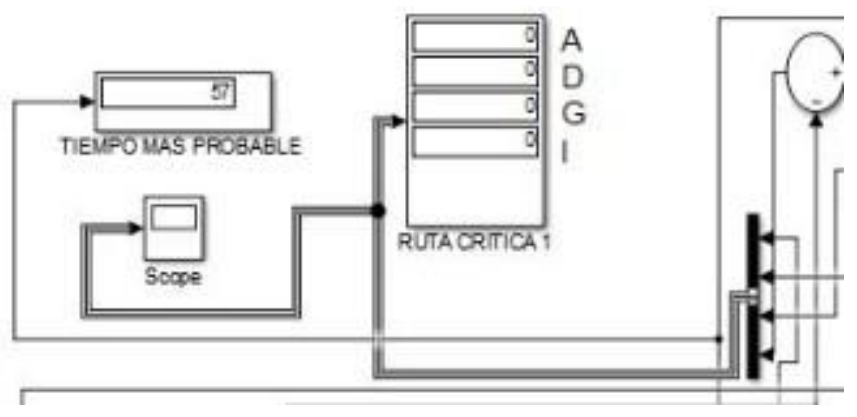

Fuente: Autor

\section{Conclusiones}

Al realizar una simulación matemática se puede optimizar el tiempo que conllevaría a cualquier gerente de proyecto la obtención de ruta crítica. Mejorando los tiempos, además de que los resultados son más exactos.

\section{Referencias Bibliográficas}

Farnum, N., \& Staton, L. (1987). Some Results Concerning the Estimation of Beta Distribution Parameters in PERT. J. Operational Res. Society. 38, 287/290.

Keefer, D. L., \& Verdini, W. A. (2015). Better Estimation of PERT Activity Time Parameters. Institute for Operations Research and the Management Sciences.

Pumnia, B., \& Khandelwal, K. (2006). Project Planning and Control with PERT and CMP. Golden House: LAXMI PUBLICATIONS.

PYME Online. (19 de 08 de 2018). Cómo realizar estimaciones de tiempo para proyectos correctas. Obtenido de http://www.pymeonline.es/2017/06/15/realizar-estimaciones-tiempoproyectos-correctas/ 
